\title{
La vérité sort de la bouche des loups. De la malédiction à la parole biblique, itinéraire spirituel d'un faux prophète sur la voie de la subversion
}

\section{Loren Gonzalez}

\section{(2) OpenEdition}

\section{Journals}

Édition électronique

URL : http://journals.openedition.org/questes/3456

DOI : 10.4000/questes.3456

ISSN : 2109-9472

Éditeur

Les Amis de Questes

\section{Édition imprimée}

Date de publication : 15 juin 2014

Pagination : 73-98

ISSN : 2102-7188

\section{Référence électronique}

Loren Gonzalez, «La vérité sort de la bouche des loups. De la malédiction à la parole biblique, itinéraire spirituel d'un faux prophète sur la voie de la subversion », Questes [En ligne], 28 | 2014, mis en ligne le 15 juin 2014, consulté le 10 décembre 2020. URL : http://journals.openedition.org/questes/3456 DOI : https://doi.org/10.4000/questes.3456 


\title{
La vérité sort de la bouche des loups. De la malédiction à la parole biblique, itinéraire spirituel d'un faux prophète sur la voie de la subversion
}

\author{
Loren GONZALEZ \\ Université de Toulouse II-Le Mirail (Laboratoire PLH-ELH)
}

Les multiples enjeux liés à la prophétie au Moyen Âge convoquent maintes figures, d'Isaïe à Merlin, en passant par les très nombreux prophètes qui ont émaillé l'histoire politique, religieuse et littéraire de 1'Occident chrétien ${ }^{1}$. À juste titre, les travaux qui leur ont été consacrés depuis plusieurs décennies sont légion; mais à l'inverse, la figure du faux prophète, laquelle représente l'envers de la prophétie et participe de sa définition même, n'a encore que peu retenu l'attention de la critique. D'une part, les figures historiques de faux prophètes nous font voyager d'Akhénathon ${ }^{2}$ à Gide ${ }^{3}$ et de Lucien de Samostate à la bande dessinée ${ }^{5}$;

\footnotetext{
${ }^{1}$ Hélène Millet, «Écoute et usage des prophéties par les prélats pendant le Grand Schisme ", dans Mélanges de l'École française de Rome. Moyen Âge, Temps modernes, 102, $\mathrm{n}^{\circ} 2$, 1990, p. 425-455. Voir également André Vauchez, «Les théologiens face aux prophéties à l'époque des papes d'Avignon et du Grand Schisme », dans Mélanges de l'École française de Rome. Moyen Âge, Temps modernes, 102, 2, 1990, p. 577-588. Voir aussi, du même auteur, Saints, prophètes et visionnaires. Le pouvoir surnaturel au Moyen Âge, Paris, Albin Michel, coll. « Bibliothèque Albin Michel de l'histoire », 1999.

${ }^{2}$ Nicholas Reeves, Akhénaton et son Dieu: pharaon et faux prophète, Paris, Éditions Autrement, coll. « Mémoires », 2004.

${ }^{3}$ André Gide, El Hadj ou Le traité du faux prophète, dans Romans : récits et soties, ouvres lyriques, Paris, Gallimard, coll. « Bibliothèque de la Pléiade », 1958.

${ }^{4}$ Lucien de Samostate, Alexandre, le faux prophète, dans Euvres choisies, éd. Jean Suret-Canale, Pantin, Le Temps des cerises, 2007.
} 
d'autre part, si l'on trouve quelques travaux récents sur ce thème dans le domaine anglo-saxon ${ }^{6}$, les références que nous avons pu répertorier au cours de nos recherches nous ont chaque fois ramenée tantôt à des ouvrages historiques engagés ${ }^{7}$, tantôt à des œuvres de fiction.

Pourtant, dès le Moyen Âge et bien avant Abraham Nelson, de nombreux clercs ont essayé de répondre à cette épineuse question ou, du moins, se sont attachés à mettre en garde leurs contemporains contre la menace évidente que représentaient les faux prophètes, tels que l'ermite Télésphore, Jean Hus ou encore Jean de Varennes ${ }^{8}$. En effet, avant même que le Grand Schisme d'Occident ne jette le trouble sur toute parole prétendument prophétique, les théologiens ont fait montre d'une véritable méfiance envers le «déferlement du discours et de l'imagerie apocalyptiques ${ }^{9} \gg$ qui assaillait les consciences, notamment à l'époque des croisades, des hérésies et des avancées arabes menées sous l'égide de la figure anti-tutélaire de Mahomet. Ainsi, d'éminents savants ont dénoncé les faux prophètes sur le modèle biblique, depuis Augustin d'Ancône ${ }^{10}$, à qui Hildegarde de Bingen ${ }^{11}$, Pierre d'Ailly ${ }^{12}$, Henri de Langenstein ${ }^{13}$ ou encore Jean Gerson ${ }^{14}$ ont ensuite emboîté le pas.

${ }^{5}$ Tarek et Vincent Pompetti, Raspoutine II : Le faux prophète, Paris, EP Éditions, coll. « Trilogies », 2007.

${ }^{6}$ James F. McGrath, « Jesus as False Prophet », dans Library of New Testament studies, 327, 2008, p. 95-110 ; Shimon Bakon, "True and False Prophets », dans Jewish Bible Quarterly, 39, 3, 2011, p. 152-158.

${ }^{7}$ Abraham Nelson, A perfect description of Antichrist, and his false prophet. Wherein is plainly shewed that Oliver Cromwell was Antichrist, and John Presbiter, or John Covenanter his false prophet, Londres, T.F., 1660.

${ }^{8}$ André Vauchez, «De l'Antiquité tardive à la fin du Moyen Âge », dans Prophètes et prophétisme, dir. André Vauchez, Paris, Éditions du Seuil, 2012, p. 112.

${ }^{9}$ Hervé Martin, Mentalités médiévales, tome II : Représentations collectives $d u X I^{e}$ au $X V^{e}$ siècle, Paris, PUF, coll. « Nouvelle Clio », 2001, p. 173.

${ }^{10}$ Augustin d'Ancône ou Agostino Trionfo, Augustini Triumphi Summa de potestate ecclesiastica, XV, 5, Rome, G. Ferrarius, 1582.

${ }^{11}$ Hildegarde de Bingen, Scivias ou Les trois livres de visions et révélations, tome I, «Vision troisième»: «ils [c'est-à-dire les païens, les hérétiques et les «pseudoprophètes »] veulent connaître ce qu'ils ne peuvent savoir, imitant celui qui voulut 
En amont, dans les Écritures, la figure du faux prophète apparaît plus d'une quinzaine de fois de l'Ancien au Nouveau Testament, avant d'être vaincue dans l'Apocalypse de Jean :

Et adprehensa est bestia et cum illo pseudopropheta qui fecitsigna coram ipso quibus seduxit eos qui acceperunt caracterem bestiae qui et adorant imaginem eius vivi missi sunt hii duo in stagnum ignis ardentis sulphure ${ }^{15}$.

Au-delà de l'image frappante, la question du lien unissant inextricablement la bête et le faux prophète mérite qu'on s'y attarde afin d'essayer de lui apporter quelque éclairage, tant elle est prégnante dans le système de représentations régissant la Vulgate, dans laquelle résonne la célèbre sentence: «Attendite a falsis prophetis, qui veniunt ad vos in

être semblable au Très-Haut. Et comme ils le suivent, il leur montre le mensonge sous l'aspect de la vérité, selon leur volonté ; c'est pourquoi ils ne sont pas avec moi, ni moi avec eux, car ils ne marchent pas dans mes voies ; mais ils aiment les sentiers détournés, recherchant ce que de folles créatures leur montrent faussement sur les causes futures » (trad. Jacques Renard, Paris, R. Chamonal, 1909).

${ }^{12}$ Pierre d'Ailly a rédigé un traité contre les faux prophètes, autour de 1380 , dans le but de dénoncer l'hypocrisie et les imposteurs à travers l'image des « loups ravisseurs », auxquels il oppose la figure authentique d'Hildegarde de Bingen. $C f$. Pierre d'Ailly, De falsis prophetis appendice dans Iohannis Gersonis opera omnia, éd. Lud Ellies Du Pin, Antwerp, Sumptibus Societatis, 1706. Voir également André Vauchez, « Les théologiens face aux prophéties à l'époque des papes d'Avignon et du Grand Schisme », art. cit., p. 580.

${ }^{13}$ Henri de Langenstein a dénoncé les prophéties de Télésphore de Cosenza et qualifié Joachim de Flore de faux prophète par excellence dans son Invectiva contra quemdam eremitom de ultimis temporibus vaticinantem nomine Telesphorum ou Liber contra vaticinici Telesphori, rédigé en 1392. Cf. Emil Donckel, «Studien über die Prophezeiung des Fr. Telesphorus von Cosenza (1365-1386)», Archivum Franciscanum Historicum, 26, 1933, p. 86-92.

${ }^{14}$ Jean Gerson a laissé deux traités sur la question, dont le De distinctione verarum visionum a falsis qui date de 1401, tandis que le De examinatione doctrinarum a été écrit à Lyon en 1423 sur le thème "Attendite a falsis prophetis». Cf. CEuvres complètes de Gerson, tome III, éd. Palémon Glorieux, Paris, Desclée, 1961, p. 36-56 et p. $458-475$.

${ }^{15}$ Apocalypse XIX, 20, Édition de la Vulgate en ligne à l'adresse suivante : http://www.thelatinlibrary.com/bible.html (ce sera notre édition de référence). Trad. Louis Segond dans La Sainte Bible, Paris, Alliance biblique universelle, 1963 (ce sera notre traduction de référence), p. 1266 : «Et la bête fut prise, et avec elle le faux prophète, qui avait fait devant elle les prodiges par lesquels il avait séduit ceux qui avaient pris la marque de la bête ». 
vestimentis ovium, intrinsecus autem sunt lupi rapaces ${ }^{16} \gg$. Dans la tradition biblique relayée par le Physiologus et les Bestiaires, le loup, ennemi du monde pastoral, est ainsi tapi dans l'ombre du mauvais berger qui laisse les brebis de l'Éternel en pâture aux faux prophètes, comme autant de bêtes sauvages dont l'imaginaire transparaît par exemple dans le Livre de Zacharie :

ecce ego suscitabo pastorem in terra qui derelicta non visitabit dispersum non quaeret et contritum non sanabit et id quod stat non enutriet et carnes pinguium comedet et ungulas eorum dissolvet ${ }^{17}$.

Dans l'imaginaire médiéval, le loup s'impose ainsi en tant qu'incarnation par excellence du faux prophète, lui qui n'est que ruse, mensonge et tromperie. Lorsque, au gré de ses péripéties littéraires, la bête immonde ouvre la bouche, ce n'est donc que pour la refermer sur quelque agneau de Dieu; et si d'aventure il se met à parler, c'est pour endormir la vigilance de sa victime et mieux la dévorer.

Néanmoins, il existe quelques textes où le loup se livre à de véritables prophéties - ou admises comme telles -, mettant ainsi à mal l'image de faux prophète qui lui colle à la peau. C'est le cas notamment dans ce prototype du Roman de Renart que constitue l'Ysengrimus de Nivard $^{18}$, où le héros éponyme transforme sa mort en une apocalypse subversive au cours de laquelle le loup se met dans la «peau» de

${ }^{16}$ Matthieu VII, 15 (éd. cit.) : « Gardez-vous des faux prophètes. Ils viennent à vous en vêtements de brebis, mais au-dedans ce sont des loups ravisseurs » (trad. cit., p. 956).

${ }^{17}$ Livre de Zacharie XI, 16, (éd. cit). «Voici que je vais faire venir dans le pays un berger qui n'aura point de souci des brebis qui périssent, qui n'ira pas à la recherche des plus jeunes, ne guérira pas celles qui sont blessées ni ne soignera celles qui sont en bonne santé ; mais il dévorera la chair des plus grasses, et il déchirera jusqu'aux cornes de leur pieds. » (trad. cit., p. 939).

${ }^{18}$ En raison des allusions politiques jalonnant les livres V à VII et de la mention d'événements survenus à Beauvais en 1150-1151, il semble très probable que le roman ait été écrit en 1152, c'est-à-dire après la deuxième croisade, mais avant la mort de Bernard de Clairvaux en 1153 ( $c f$. Elisabeth Charbonnier, Recherches sur l'Ysengrimus : traduction et étude littéraire, "Introduction », thèse imprimée, Université Paris III-Sorbonne Nouvelle, 1982, tome I, p. 14-15). 
l'évangéliste Jean pour rejouer les différentes scènes de ce texte ultime, de la dévoration du Livre de la Paix au cataclysme final, censé marquer la victoire sur les faux prophètes (Apocalypse XIX, 11). Mais au-delà de la figure tutélaire d'Ysengrin, le loup littéraire du XII siècle est bien souvent ce « retourne-peau », le versipellis ou loup-garou qui peut à chaque instant changer de forme, voire de faux prophète devenir messager des vérités divines. C'est d'ailleurs l'hypothèse émise dans la seconde partie de la Topographia Hibernica de Giraud de Barri (1188), florilège d'anecdotes sur l'histoire du pays et au chapitre XIX duquel nous est relatée, à la première personne, la rencontre entre un prêtre et un loup-garou très pieux ; tout droit sorti des fantasmes générés par l'Irlande chez les clercs du $\mathrm{XII}^{\mathrm{e}}$ siècle, celui-ci intercepte le prêtre en pleine forêt afin qu'il donne l'extrême-onction à sa compagne, près de mourir dans sa pelisse de louve ; après quoi, il prophétise sur l'avenir de l'Irlande ${ }^{19}$.

Entre mirabilis et roman satirique, incarnation du septénaire des vices et garou repentant, Ysengrimus et la Topographia Hibernica, deux œuvres latines de la seconde moitié du XII ${ }^{\mathrm{e}}$ siècle fortement imprégnés par la culture cléricale, interrogent chacune à leur façon les limites de la prophétie médiévale en imposant au discours prophétique, en surimpression, toute la charge subversive qui peut sortir de la bouche d'un loup. L'éclairage apporté par ces deux textes nous aidera donc peut-être à comprendre comment le loup, incarnation du faux prophète, en vient à convertir sa parole subversive en vérité chrétienne. Dans un contexte de mort imminente, où la bête et l'expression littéraire s'épuisent dans un même mouvement, l'on tentera ainsi de retracer l'itinéraire de ces faux

${ }^{19}$ Giraldus Cambrensis, Topographia Hibernica, II, 19, dans Euvres complètes, éd. James Francis Dimock, London, Longmans, 1867, p. 101-106. Cf. également Jeanne-Marie Boivin, L'Irlande au Moyen Âge. Giraud de Barri et la Topographia Hibernica, Paris, Champion, coll. « Nouvelle Bibliothèque du Moyen Âge », 1993, p. 211-215. 
prophètes vers la vérité en interrogeant d'abord leur qualité de faux prophète, puis leur cheminement vers l'acte prophétique, avant d'en dégager la portée subversive.

\section{Les faux prophètes, du loup au loup-garou}

\section{La figure du faux prophète, de la tradition vétérotestamentaire au Moyen Âge chrétien}

Le prophète est «celui qui comprend ${ }^{20}$ » et interprète le cours de l'Histoire, grâce à l'omniscience conférée par ses cinq vertus « cardinales » qui sont, selon Jean Gerson ${ }^{21}$, l'humilité, la discrétion, la patience, l'amour de la vérité et la charité22.

Quant au faux prophète, il est menteur et démagogue sous la loi de Moïse, annonçant que tout ira bien, comme dans le Livre de Jérémie : « et curabant contritionem filiae populi mei cum ignominia dicentes pax pax et non erat pax $^{23} \gg$ (Jérémie, VI, 14). D'ailleurs, on prête volontiers attention à ses discours hypocrites: "utinam non essem vir habens spiritum et mendacium potius loquerer stillabo tibi in vinum et in ebrietatem et erit super quem stillatur populus iste ${ }^{24} \gg$ (Michée II, 11). Précisons également que, suivant toute logique, la prophétie du faux prophète ne s'accomplit jamais $^{25}$, ce qui n'empêche pas la prolifération de ces imposteurs, dont le

${ }^{20}$ Ursula Fortiz, Prophètes et prophéties : histoires et tradition, Paris, De Vecchi, 2004, p. 185.

${ }^{21}$ Jean Gerson, De distinctione verarum visionum a falsis, dans CEuvres complètes de Gerson, tome III, éd. cit., p. 38.

${ }^{22}$ André Vauchez, «De l'Antiquité tardive à la fin du Moyen Âge », art. cit., p. 113 : «À ses [Gerson] yeux, cinq vertus étaient nécessaires chez le prophète : l'humilité, la discrétion, la patience, l'amour de la vérité et la charité. ».

23 « Paix, paix ! disent-ils. Et il n’y a point de paix » (trad. cit., p. 752).

${ }^{24}$ «Si un homme court après le vent et débite des mensonges : "Je vais te prophétiser sur le vin, sur les boisons fortes !", ce sera pour ce peuple un prophète. » (trad. Louis Segond p. 918).

${ }^{25}$ Jérémie (Jé. XXVIII, 9) prévient ainsi que «propheta qui vaticinatus est pacem cum venerit verbum eius scietur propheta quem misit Dominus in veritate ». "C'est par l'accomplissement de ce qu'il prophétise qu'il sera reconnu comme véritablement 
nombre atteint quatre cents dans le Livre des Rois (1Rois XXII, 6). Enfin, le faux prophète usurpant l'autorité de Dieu est traditionnellement assimilé au païen ou à l'infidèle "propheta autem qui arrogantia depravatus voluerit loqui in nomine meo quae ego non praecepi illi ut diceret aut ex nomine alienorum deorum interficietur ${ }^{26} »$ (Deutéronome XVIII, 20).

Parce que le faux prophète incarne l'autre, celui qui porte la parole de l'autre religion, l'on comprend ainsi que, pour le Moyen Âge des croisades et des conquêtes musulmanes, le faux prophète par excellence n'est autre que Mahomet, auquel s'identifie Ysengrin au vers 295 du roman éponyme, au moment où, précisément, il annonce qu'il va prophétiser: «Mors Mahamet patienda michi est ${ }^{27}$ ! ». Mais pour saisir les enjeux et les raisons de cette filiation assumée, il nous faut examiner de plus près les représentations du loup qui ont marqué la symbolique médiévale.

\section{Le loup, faux prophète par excellence}

En tant que figure privilégiée de l'hérésie, incarnation de la bête féroce du paganisme ${ }^{28}$, animal des temps barbares d'avant la Grâce, le loup s'impose de toute évidence comme le double privilégié du faux prophète au Moyen Âge. De surcroît, ce missionnaire du chaos est l'antithèse du bon berger de la Bible, lequel présente un certain nombre de points communs avec le prophète puisqu'il est, comme lui, un guide capable de rassembler

envoyé par l'Éternel» (trad. cit., p. 779 ). Cf. aussi Deutéronome XVIII, 22 : « hoc habebis signum quod in nomine Domini propheta ille praedixerit et non evenerit hoc Dominus non locutus est sed per tumorem animi sui propheta confinxit et idcirco non timebis eum ». «Quand ce que dira le prophète n'aura pas lieu et n'arrivera pas, ce sera une parole que l'Eternel n'aura point dite » (trad. cit., p. 212).

${ }^{26}$ « Le prophète qui aura l'audace de dire en mon nom une parole que je ne lui aurai point commandée ou qui parlera au nom d'autres dieux, ce prophète-là sera puni de mort. » (trad. cit., p. 212).

${ }^{27}$ «Il me faut mourir comme Mahomet !» (trad. Élisabeth Charbonnier, thèse citée. Ce sera notre traduction de référence).

${ }^{28}$ L'on verra combien le passage du paganisme au christianisme, voire le syncrétisme, sont essentiels dans le cadre de cette réflexion. 
les fidèles et dont la parole ou la voix jouent un rôle éminemment symbolique : en effet, le vrai prophète aussi «domestique» les foules ${ }^{29}$, tandis que le mauvais berger qui conduit mal son troupeau le livre aux loups ravisseurs ${ }^{30}$.

Cependant, au Moyen Âge, le loup est surtout la bête mordicante, cette gueule d'Enfer prête à dévorer le monde, gueule avide et insatiable qui sème la mort et dégouline du sang des innocents. Pourtant, la gueule du loup n'est pas simplement le « lieu de la préhension de la proie et de la dévoration, mais aussi l'organe de la parole humaine ${ }^{31} »$. Précisément, le loup entretient un rapport étroit avec la parole, la voix et le souffle même, ce qui fait de lui le réceptacle privilégié d'un questionnement sur la bonne ou la mauvaise parole, la parole séductrice, celle qui ravit : la prophétie en somme, qui est avant tout un acte de parole. En effet, nombreuses sont les croyances selon lesquelles le loup ôte la voix à l'homme rencontré par hasard, si par malheur celui-ci est découvert en premier par la bête : «Et si est [la louve] de tel nature que s'ele puet sor un home alener et veïr sa bouce ovrir, il en pert sa vois ${ }^{32} »$, lit-on par exemple dans la version longue

${ }^{29}$ Henri Martin, Mentalités médiévales, tome II : Représentations collectives $d u X I^{e}$ au $X V^{e}$ siècle, Paris, PUF, coll. « Nouvelle Clio», 2001, p. 193. Cf. également Ézéchiel XXXIV, 11-16 et surtout 11-13 : « et educam eas de populis et congregabo eas de terris et inducam eas in terram suam et pascam eas in montibus Israhel »; «je les retirerai d'entre les peuples, je les rassemblerai des diverses contrées, et je les ramènerai dans leur pays ; je les ferai paître sur les montagnes d'Israël » (trad. cit., p. 858).

${ }^{30} \mathrm{Cf}$. par exemple Zacharie XI, 17 : «o pastor et idolum derelinquens gregem ». « Malheur au pasteur de néant, qui abandonne ses brebis » (trad. cit., p. 939).

${ }^{31}$ Roger Bellon, «Variations sur un couple. Le loup et le Renard de l'Ysengrimus au Renart français ", dans Le rire de Goupil : Renard, prince de l'entre-deux, Toulouse, Le Tournefeuille, Librairie Jouannaud, 1998, p. 66.

${ }^{32}$ Le Bestiaire : version longue attribuée à Pierre de Beauvais, éd. Craig Baker, Paris, Champion, coll. « Classiques français du Moyen âge », 2010, p. 225. 
du Bestiaire attribuée à Pierre de Beauvais, comme chez Richard de Fournival ${ }^{33}$ ou dans l'œuvre de Galand de Reigny ${ }^{34}$.

Selon Claude Gaignebet, cette croyance ne se rapporte pas tant à la peur paralysante qu'inspire le loup qu'à son pouvoir «lieur ${ }^{35} »$ : en effet, le loup lie et noue les gorges ${ }^{36}$, tandis que celui qui les dénoue et rend la parole aux hommes n'est autre que saint Blaise, dont le nom vient du loup («bleid» en breton) et qui a le pouvoir de guérir les gorges malades : « le loup saisit la voix parce qu'il détient un pouvoir magique lieur. Saint Blaise, autrement dit le "loup" chrétien, possède justement le pouvoir de délier la gorge en opérant à l'inverse du dieu-loup qu'il christianise ${ }^{37}$ ». Par ailleurs, si le loup et Blaise ${ }^{38}$ ne sont peut-être que les deux facettes d'une même figure, Blaise est aussi, selon Philippe Walter, le «jumeau mythique » de Merlin ${ }^{39}$, de même que le loup constitue le double sauvage

${ }^{33}$ Richard de Fournival, Le Bestiaire d'amour et La Response du bestiaire, éd. Gabriel Bianciotto, Paris, Champion, coll. «Champion classiques », 2009, p. 164 : « puis que je fui premerains veüs, selonc le nature du leu, j'en dui bien perdre le vois ». $C f$. également Le Bestiaire de Cambrai, éd. Jean-François Kosta-Théfaine, Rouen, C. Chomant, coll. «Textes anciens », 2009, p. 76: «La nature dou leu si est que, quant il voit premiers .i. home anchois que li hons le voie, li hons en pert de se forche et de se vois et converso $\gg$.

${ }^{34}$ Galand de Reigny, Petit livre de proverbes, éd. Jean Châtillon, Maurice Dumontier et Alexis Grélois, Paris, Éditions du Cerf, coll. « Sources chrétiennes », 1998, proverbe 133 : "Porro lupum ouibus superuenientem, si fieri queat, ante ipse uidere quam ab ipso uideatur debet, ne si forte prior uisus fuerit, uocis sono repente amisso, contra eum uociferari iam non sufficiat." («Enfin, quand le loup fond sur les brebis, le berger doit, autant que possible, le voir avant d'être vu, de peur, si d'aventure il est vu le premier, de perdre soudain la voix et de se trouver hors d'état de crier après lui. », trad. des éditeurs).

${ }^{35}$ Claude Gaignebet, Le Folklore obscène des enfants, Paris, Maisonneuve et Larose, 1980, p. 55.

${ }^{36}$ On dit d'ailleurs de celui qui est enroué qu'il a vu le loup.

${ }^{37}$ Philippe Walter, Merlin ou le savoir du monde, Paris, Imago, 2000, p. 61. Pour plus de précisions, $c f$. également, du même auteur, dans ibid., le chapitre intitulé «Le loup et la voix », p. 59-64.

${ }^{38}$ Pour un dossier complet au sujet de saint Blaise, voir Claude Gaignebet, A plus hault sens : l'ésotérisme spirituel et charnel de Rabelais, Paris, Maisonneuve et Larose, 1986, p. 41-51.

${ }^{39}$ Philippe Walter, Merlin ou le savoir du monde, op. cit., p. 64-65. 
du prophète dans la Vita Merlini ${ }^{40}$. En vertu de cette double filiation, le loup s'impose inéluctablement comme agent de parole dans l'imaginaire médiéval, capable de donner ou d'ôter la faculté de parler, capable aussi bien de mentir que de dire la vérité. Tout à la fois instrument de la satire et de la prophétie, le loup fait ainsi basculer les enjeux de l'œuvre littéraire dès lors que dans l'espace textuel, il fait fusionner ces deux discours dans sa gueule d'enfer.

\section{Ysengrin et le loup-garou d'Ossory : de vrais faux prophètes}

L'Ysengrin de Nivard a tout du faux prophète : stultus senex, il n'a pas a priori cette connaissance supérieure qui est la marque des vrais prophètes. Incapable de respecter la parole donnée, c'est un parjure assumé et un menteur, qui ne plaide pour sa bonne foi que pour mieux endormir la vigilance de ses victimes potentielles ${ }^{41}$. Mauvais berger lorsqu'il se mêle d'occuper quelque fonction pastorale, c'est un véritable antimoine ${ }^{42}$ qui

${ }^{40}$ Geoffroy de Monmouth, Vita Merlini, dans Le devin maudit. Merlin, Lailoken, Suibhne, dir. Philippe Walter, Grenoble, ELLUG, 1999, coll. « Moyen Âge européen », v. 102-104, p. 62-64: "Tu lupe care comes, nemorum qui devia mecum / et saltus peragrare soles vix preteris arva, / et te dura fames et me languere coegit» (trad. en regard par Philippe Walter, p. 63-65 : « Toi, loup, cher compagnon, qui parcours d'habitude les chemins détournée des bois et des breuils en ma compagnie, c'est avec peine que tu traverses les champs : la faim cruelle nous réduit tous deux à languir ». Le tutoiement, les termes affectifs, tel « care », la présence en fin de l'hexasyllabe de l'enclise «mecum », ainsi que le parallélisme de construction opéré au vers 104 autour d'un balancement entre les pronoms de la première et de la deuxième personne, se font l'écho de véritables liens d'amitié unissant Merlin à ce loup mystérieux, présenté au détour d'une exclamation poétique. L'affection est tangible de la part du personnage à l'égard de l'animal qui n'est "guère maintenu dans l'altérité et semble confondre ses traits avec ceux du fou » (Philippe Walter, Merlin ou le savoir du monde, op. cit., p. 21).

${ }^{41}$ Nivardus, Ysengrimus, éd. Ernst Voigt, Hildesheim, New York, Olms, 1974, Livre V, v. 581-582, p. 291 : « Explorate fidem, quam uobis spondeo, fraters, / Ars mea, quod fertis, grande leuabit onus » («Mettez à l'épreuve la parole que je vous engage, mes frères, mon habileté allègera le lourd fardeau que vous portez », trad. cit.).

${ }^{42}$ Le loup représente en effet l'antimoine dans la symbolique alchimique, qualifiée de lupus metallorum (« loup des métaux » ou « loup dévorant des métaux »). L'image est par exemple convoquée dans la première des Douze clefs de la philosophie de Basile Valentin (éd. Eugène Canseliet, Paris, Éditions de Minuit, 1992) et dans le 
parodie l'univers clérical rongé par le vice et dont Nivard nous brosse un tableau sans concession. Mais l'Ysengrimus est un roman essentiellement parodique où tout sonne faux, jusqu'à l'apocalypse du livre VII dans lequel tout part à vau-l'eau, le fil du récit se laissant emporter dans cette étrange logorrhée prophétique que vomit le loup dans son dernier souffle, et qu'il dédie au démon païen Agemundus, lutin ou feu follet farceur, "né d'une tradition folklorique mitigée de symbolique chrétienne ${ }^{43} »$ voire, de tout un bestiaire :

uerum fortuna prophetam

Illius auxilio demonis esse dedi

Cuius ut accipitris rostrum, iuba sicut equina est,

Catti cauda, bouis cornua, barba capre,

Lana tegit lumbos, dorsum plumatur ut anser, Ante pedes galli, post habet ille canis ${ }^{44}$.

Le problème du paganisme se pose aussi dans le mirabilis présenté par Giraud de Barri, où l'on ne sait pas trop bien si le loup-garou d'Ossory est une créature païenne ou l'œuvre étrange de Dieu. Faux prophète ou prophète en faux? Le garou avoue que son peuple a été maudit pour ses péchés, probablement liés à l'adoration des idoles, laquelle avait encore cours en Irlande au XII ${ }^{\mathrm{e}}$ siècle ${ }^{45}$.

De quodam hominum genere sumus Ossiriensum. Unde, quolibet septennio, per imprecationem sancti cujusdam, Natalis scilicet abbatis, duo, videlicetmas et femina, tam a formis quam finibus

Splendor Solis de Salomon Trismosin (éd. Jörg Völlnagel, Berlin, Dt. Kunstverl, 2004).

${ }^{43}$ Elisabeth Charbonnier, Recherches sur l'Ysengrimus..., thèse citée, tome III, p. 263, note 24 .

${ }^{44}$ Nivardus, Ysengrimus, éd. Ernst Voigt, op. cit., v. 365-368, p. 387 ; « la Fortune lui avait vraiment permis de prophétiser grâce à l'aide de ce démon qui a un bec d'épervier, une crinière de cheval, une queue de chat, des cornes de bœuf, une barbe de chèvre ; de la laine couvre ses reins, mais il a le dos plein de plumes comme un jars. Devant, il a les pattes d'un coq, derrière celles d'un chien. » (trad. cit.).

${ }^{45}$ L'on verra que la morale attachée à la prophétie du loup porte précisément sur ce contexte religieux particulier, où le paganisme opérait un retour en force sur l'île. 


\section{exulare coguntur. Formam enim humanam} prorsus exuentes, induunt lupinam ${ }^{46}$.

Le personnage ne cherche d'ailleurs point à dissimuler ce péché originel mais il explique calmement que sa forme de loup lui vient de la pénitence imposée par $\mathrm{Natal}^{47}$, faisant ainsi montre de cette qualité essentielle du vrai prophète qu'est l'amour de la vérité. Mais plus encore, ce loup-garou rassemble en lui tous les caractères du bon prophète : il est charitable, puisqu'il prend des risques afin que sa compagne puisse mourir dignement, il est humble face aux péchés de son peuple, et discret: en effet, ni le prêtre ni son serviteur ne l'entendent approcher jusqu'à temps qu'il soit face à eux («ecce lupus ad eos accedens $\left.{ }^{48} »\right)$. Plus loin, il disparaît même quelques instants sans que personne ne s'en aperçoive («lupus qui parumper abscesserat $\left.{ }^{49} »\right)$; enfin, il se montre patient face aux hésitations du prêtre, le ménage et expose calmement son problème ${ }^{50}$. Il a donc tout du prophète, si ce n'est l'aspect ; mais là réside tout l'enjeu de cet épisode qui invite le lecteur à chercher la vérité derrière de sombres apparences. Pour ce faire, encore faut-il se poser plus précisément la question des enjeux attachés aux prophéties des loups, afin d'en déterminer la nature et la portée.

${ }^{46}$ Giraldus Cambrensis, Topographia Hibernica, II, XIX, op. cit., p. 102 ; «Nous sommes originaires d'Ossory. Notre peuple, maudit par le saint abbé Natal, doit tous les sept ans bannir deux individus, un homme et une femme, tant par leur forme que de leur patrie : ils se dépouillent entièrement de leur forme humaine pour revêtir celle des loups. " (trad. Jeanne-Marie Boivin dans L'Irlande au Moyen Age..., op. cit., p. 211. Ce sera notre traduction de référence).

${ }^{47}$ Notons par ailleurs que le nom de Natalis renvoie certes à la fête de Noël, mais surtout à la croyance selon laquelle les hommes nés à Noël deviennent loups (cf. ibid., p. 327, note 4).

${ }^{48}$ Giraldus Cambrensis, Topographia Hibernica, éd. cit., p. 101 ; « voici que s'approche d'eux un loup » (trad. cit., p. 211).

${ }^{49}$ Ibid., p. 102 ; « Alors le loup, qui s’était momentanément éloigné» (trad. cit., p. 212).

${ }^{50}$ Nous verrons cependant dans la suite de l'étude qu'à un moment très important du texte, le loup perd quelque peu patience et prend alors l'ascendant sur le prêtre. 


\section{Du faux prophète à la prophétie : un itinéraire ascendant sur le chemin des loups}

\section{L'acte prophétique en contexte}

Dans le récit de Giraud de Barri, la scène se passe en pleine nuit au cœur d'une forêt du Meath, dans ce même comté où se trouve la colline de Tara, haut lieu du paganisme celtique ${ }^{51}$. D'emblée, le prêtre - comme le lecteur - est réticent face à ce loup qui vient à lui, doué de parole contrairement à la plupart des loups-garous de la littérature médiévale. C'est donc par des propos doux et raisonnables - «verba de Deo sana ${ }^{52} »-$ que la créature parvient à convaincre le prêtre de sa bonne foi : «catholicum in omnibus responsum praebens ${ }^{53} »$. Mais au moment d'accorder le viatique, la scène bascule et le loup, faisant face aux hésitations du prêtre, prend soudain le contrôle pour orchestrer le rituel à sa place, en lui désignant notamment le livre liturgique et les hosties :

Lupus qui parumper abscesserat iterum accessit, ostendens ei perulam, librum manualem et aliquot hostias consecratas continentem ${ }^{54}$;

Entre brusquerie instinctive, donc animale, et geste liturgique, les questions fusent et la confusion règne au seuil du moment prophétique, notamment dans l'Ysengrimus où, comme dans la prophétie, se mêlent et s'assimilent le passé, le présent et l'avenir, le temps de la fable, celui de

${ }^{51}$ Sur ce thème, cf. Barry Raftery, L'Irlande celtique avant l'ère chrétienne, Paris, Éditions Errance, 2006 ; voir également Christian-J. Guyonvarc'h et Françoise Le Roux, La Civilisation celtique, Rennes, Ouest-France Université, coll. « De mémoire d'homme : l'histoire », 1990.

${ }^{52}$ Giraldus Cambrensis, Topographia Hibernica, II, 19, op. cit., p. 101 ; « des paroles sensées, inspirées de Dieu » (traduction préconisée par les éditrices).

${ }^{53}$ Ibid., p. 101 ; «il répondit en tous points comme un bon catholique » (trad. cit., p. 212).

${ }^{54}$ Giraldus Cambrensis, Topographia Hibernica, II, XIX, op. cit., p. 102 ; «Alors le loup, qui s'était momentanément éloigné, revint et lui désigna une petite besace contenant un livre liturgique et quelques hosties consacrées » (trad. cit., p. 212). 
l'Histoire et celui de l'eschatologie ${ }^{55}$. Ainsi le dernier livre, le plus original et d'ailleurs jamais repris par la tradition renardienne, est-il conçu comme une réécriture de l'Apocalypse de Jean où Ysengrin joue tous les rôles, celui de la Bête, celui du faux prophète vaincu et celui du témoin visionnaire. Cependant, en cette fin de roman, le récit s'affole et la mise en scène boîte comme notre loup à qui il manque une patte ${ }^{56}:$ les anges sont remplacés par des porcs qui chantent faux, la voix céleste n'est autre que celle de la truie Salaura, marraine et bourreau d'Ysengrin, les trompettes sont des clochettes, et le Livre de Paix qu'avale Jean au verset X, 10 de l'Apocalypse est ici un traité de paix, que le loup aurait avalé dans un horstexte fantasmatique.

Germana Sonoche, Aspice, quod fecit perfidus iste nefas! Glutierat librum, quo pax oblata daretur, Et michi latorem se fore pacis ait ${ }^{57}$ !

Mais une fois le livre retrouvé dans le foie ${ }^{58} \mathrm{~d}^{1}$ Ysengrin, cruellement arraché, le loup apparaît comme transfiguré : l'intuition de son agonie prochaine génère l'assimilation à Mahomet, dévoré par des porcs dans le

${ }^{55}$ Il s'agit en effet d'un texte historiquement très marqué, d'où sa datation assez précise aux alentours de 1152 (cf. infra, note 18).

${ }^{56}$ Nivardus, Ysengrimus, éd. Ernst Voigt, op. cit., v. 15, p. 366 : « pedis unium orbum » (« avec une patte en moins », trad. cit.). Cette claudication n'est pas anodine, si l'on songe que, selon le savant juif Maïmonide, « lorsqu'ils [les prophètes] prophétisent, leurs membres tremblent, leur force chancelle » ( $c f$. Moïse Maïmonide, Le Livre de la Connaissance, trad. Valentin Nikiprowetzky et André Zaoui, Paris, PUF, 1961, chapitre VII, p. 85). Nous signalons que le texte original en hébreu de cet ouvrage fondamental est disponible dans l'édition Rubinstein-Rabinowicz, et qu'il existe une édition latine, laquelle ne fait cependant pas autorité : De fundamentis legis, éd. Guil Vorstius, Amsterdam, apud Guiliel \& Iohannem Blaeu, 1638.

${ }^{57}$ Nivardus, Ysengrimus, éd. Ernst Voigt, op. cit., v. 287-290, p. 382 ; « Sonoche, ma sœur, vois ce qu'a fait ce traître criminel ! Il avait avalé le livre contenant l'offre de paix, et il me dit qu'il apporte la paix ! » (trad. cit.).

${ }^{58}$ Il semble d'ailleurs très intéressant que le Livre soit précisément extrait du foie d'Ysengrin, siège des passions dans la symbolique médiévale. En effet, Ysengrin est dépeint dans le roman comme un personnage tout entier guidé par la folie de ses passions, folie dont on rappelle qu'elle n'est pas étrangère à l'acte prophétique. Ainsi le choix de cet organe participe-t-il avec une habileté certaine du caractère proprement ambigu de la position d'Ysengrin en tant que faux prophète. 
désert $^{59}$, et déclenche en cascade la logorrhée prophétique. Le lecteur est donc averti qu'il s'agira d'une fausse prophétie puisqu'elle se place d'emblée sous le signe du faux prophète par excellence, Mahomet. Mais alors que dans l'Apocalypse, Jean est exhorté à prophétiser après avoir avalé le Livre de Paix - «et dicunt mihi oportet te iterum prophetare ${ }^{60} »$ (Apocalypse X, 11) - c'est ici le contraire et Ysengrin est obligé de prévenir qu'il va prophétiser, ce qui l'enjoint à imiter non seulement le prophète mais aussi la voix qui s'adresse à Jean dans l'Apocalypse :

Cedite dumtaxat, donec uentura prophetem.

Effugere amisi, cedite queso parum!

Ceditur, ille canit, plaudit fortuna canenti ${ }^{61}$

Le syntagme (verbe + complément) « ventura prophetem » intervient en premier, puis le verbe canere est répété deux fois au vers 299. Ce terme, qui signifie avant tout « chanter » ou jouer d'un instrument de musique, ne va d'ailleurs pas sans évoquer l'extase comme forme première de la prophétie $^{62}$ selon Isidore de Séville, ainsi que la cinquième forme qui fait appel à une « voix céleste ${ }^{63} »$.

\section{Nature et portée de la prophétie du loup}

Ici, nulle extase pourtant, sinon une authentique prophétie de malédiction qui condamne l'humanité toute entière, à l'exception des rares

59 Embricon de Mayence, Vita Mahumeti [milieu du XI $\mathrm{XI}^{\mathrm{e}}$ siècle], éd. Guy Cambier, Bruxelles-Berchem, Latomus Revue d'études latines, 1962.

${ }^{60}$ « Puis on me dit : il faut que tu prophétises de nouveau » (trad. cit., p. 1258).

${ }^{61}$ Nivardus, Ysengrimus, éd. Ernst Voigt, op. cit., v. 297-299, p. 382 ; «Reculez, le temps pour moi de prédire l'avenir, j'ai renoncé à fuir, je vous en prie, reculez un peu ! On recule, il prophétise, et la Fortune applaudit à ses prophéties » (trad. cit.).

${ }^{62}$ Isidore de Séville, Étymologies, Livre VII : Dieu, les anges, les saints, 8, 33, éd. JeanYves Guillaumin, Paris, Les Belles Lettres, coll. « Auteurs latins du Moyen Âge », 2012, p. 115 : «Prophetiae autem genera septem sunt. Primum genus extasis, quod est mentis excessus »; trad. Jean-Yves Guillaumin et Pierre Monat dans ibid, p. 114 : « Il y a sept genres de prophétie. Le premier est l'extasis ("état d'inconscience »), c'est-à-dire la perte de conscience ».

${ }^{63}$ Ibid., 8, 35, p. 117 : «Quintum genus uox de caelo»; trad. cit., p. 116 : « Le cinquième est la voix venue du ciel ». 
femmes vertueuses. Ysengrin maudit toutes les autres, semant ainsi le chaos dans l'imaginaire du roman à travers la représentation hystérique de chaises renversées et autres signes de désordre ménager. Dès lors, le texte parodique s'affranchit de la tutelle de Jean et propulse le lecteur dans un cadre très familier pour proposer sa propre apocalypse, d'une trivialité déroutante :

In lare quodcumque est ustensile sine supellex

Esto uagans, sparsim singula iacta cubent :

Straba supineturn transuersa cathedra iaceto

Prodeat aut redeat sospite nemo genu ;

Urceus, olla, lebes, coclear, lanx, peluis, aenum,/

Scrutaque diuerse sparsa uagentur opis,

Integra mane, eadem sint uespere fiss $a^{64}$;

La part de la malédiction, assortie à la perspective du trépas, est aussi très forte dans la Topographia Hibernica, puisque le loup est une créature maudite qui se met à prophétiser après la mort de son double féminin. Par contre, son portentum tient plutôt de l'exégèse, elle-même consacrée en tant qu'acte prophétique au Moyen Âge ${ }^{65}$. En effet, la prophétie du loupgarou s'inspire du Lévitique (Lv. XVIII, 27-2866) et de l'Ecclésiastique (Ecl. $\mathrm{x}, 8)$, lesquels s'inscrivent dans le texte presque à la manière d'un palimpseste ou de strates exégétiques, puisque ces références n'ont vraisemblablement été ajoutées au corps du texte qu'à partir de la

${ }^{64}$ Nivardus, Ysengrimus, éd. Ernst Voigt, op. cit., v. 354-359, p. 386-387 ; « Alors, que tous les ustensiles de la maison se promènent ici et là, que tout soit jeté par terre au hasard: le tabouret aura les pieds en l'air, la chaise sera renversée, personne n'avancera ni ne reculera sans se faire mal aux genoux : la cruche, la marmite, le chaudron, la cuiller, le plat, la bassine, le fait-tout, et diverses vieilles hardes se promèneront, répandues au petit bonheur. » (trad. cit).

${ }^{65}$ Voir à ce sujet l'article de Valeria Ingegno, infra, p. 29-52.

${ }^{66}$ Notons d'ailleurs que le Lévitique est le livre des lois et des sacrifices, des bénédictions et des malédictions. Dans le contexte du verset qui nous intéresse, il est ainsi question du tabou de bestialité (Lv. XVIII, 23), ce qui n'est pas sans rapport avec le questionnement en jeu ici, à savoir le brouillage des frontières entre les règnes humain et animal. 
quatrième édition ${ }^{67}$. En même temps, cette prophétie propose une lecture moralisée de l'Histoire, l'invasion anglaise étant comprise par le loup comme un châtiment divin à l'encontre de l'Irlande retombée dans le paganisme :

Cui lupuis: "Propter peccata", inquit, "populi nostri, et vitiorum enormitates, ira Dei descendens in generationem pravam, dedit eam in manus inimicorum. Quamdiu ergo gens ea mandata Domini custodierit, et in viia ejus ambulaverit, tuta manebit et inconvulsa. Sin autem, quia proclivis est cursus ad voluptates, et imitatrix natura vitiorum, ad nostros ex convictu mores forte descenderit, divinam in se quoque proculdubio vindictam provocabunt ${ }^{68}$.

Dans ce climat, la prophétie se charge d'une double destination, s'adressant non pas tant aux Irlandais qu'aux Anglais et à tous les autres peuples de la Chrétienté, pressés d'affermir leur foi sous peine de subir à leur tour la colère de Dieu. Comme dans Ysengrimus, les temporalités se chevauchent, le temps de la fable étant suspendu à ce moment du texte pour laisser s'interpénétrer les temps historique et biblique. L'on peut dès lors s'interroger quant à la teneur de cette vérité biblique émanant de la bouche d'un loup.

${ }^{67}$ Jeanne-Marie Boivin, L'Irlande au Moyen Âge...op. cit., p. 328, note $8:$ « Ce passage [...] n'apparaît qu'à partir de ce que Dimock considère comme la quatrième version de l'œuvre. Il témoigne du goût de Giraud pour les portenta et particulièrement pour ceux qui sont liés à la conquête de l'Irlande ».

${ }^{68}$ Giraldus Cambrensis, Topographia Hibernica, II, XIX, op. cit., p. 103 ; " "C'est", répondit le loup, "à cause des péchés et des vices monstrueux de notre peuple que la colère de Dieu s'est abattue sur une génération mauvaise et l'a livrée aux mains des ennemis. Aussi longtemps que la nation étrangère observera les commandements du Seigneur et marchera dans Ses voies, elle sera en sûreté et restera invincible. Mais si - la pente des plaisirs étant aisée et la nature prompte à imiter les vices - ils en viennent, à notre contact, à adopter nos mœurs dépravées, sur eux aussi sans doute ils attireront la vengeance divine » (trad. cit., p. 213). 
Un itinéraire ascendant vers la prophétie : conversion ou subversion?

En réalité, nos deux prophètes sont mus par une dynamique ascendante en vertu de laquelle ils évoluent et se métamorphosent au rythme du récit, des mots et de la progression du sens. D'ailleurs, comme si l'ouverture sur 1'Autre Monde conférait à nos faux prophètes un savoir inaccessible au commun des mortels, l'acte prophétique annonce, aussi bien chez Nivard que dans le texte de Giraud de Barri, la fin d'une vie, entre agonie apocalyptique et derniers sacrements; mais la prophétie signifie également la fin du récit, puisque les deux textes culminent et se terminent avec l'acte prophétique. En effet, la narration du mirabilis irlandais s'achève sur la prophétie du loup pour se transformer en réflexion théologique sur la métamorphose, après que le garou a fait ses preuves en tant qu'animal-guide : «lupum praecedentem ${ }^{69} »$, «presbyter sequitur ${ }^{70}$, «ipsos conducens ${ }^{71} »$, « viam praeostendit ${ }^{72} »$.

Quant au livre VII de l'Ysengrimus, il clôt le roman sur une apothéose parodique, pour faire de cette fin un feu d'artifice verbal imitant l'Apocalypse de Jean. Nos deux textes vont ainsi crescendo jusqu'à la prophétie finale, notamment dans la Topographia Hibernica où l'on assiste à la conversion du loup-garou, créature païenne donc démoniaque qui erre dans la forêt irlandaise - temple du paganisme au XII ${ }^{\mathrm{e}}$ siècle $^{73}$ - avant de révéler sa foi chrétienne à travers ses paroles, sa connaissance des rites et son discours prophétique. L'extrême-onction de sa compagne symboliserait-elle en même temps le baptême du loup-garou ? Cela expliquerait

\footnotetext{
${ }^{69}$ Giraldus Cambrensis, Topographia Hibernica, II, 19, op. cit., p. 102 ; « le loup passa devant » (trad. cit., p. 212).

${ }^{70}$ Ibid., p. 102 ; « le prêtre le suivit » (trad. cit., p. 212).

${ }^{71}$ Ibid., p. 103 ; « il les conduisit» (trad. cit., p. 212).

${ }^{72}$ Ibid., p. 103 ; « montra le chemin » (trad. cit., p. 212).

${ }^{73}$ Surtout dans l'esprit des clercs chrétiens.
} 
que le personnage manifeste autant de ferveur et justifierait même qu'il puisse aspirer, enfin, au statut de vrai prophète.

\section{Implications de la vérité dans le discours prophétique : lorsque « la vérité sort de la bouche des loups»}

Si la progression des loups vers la vérité prophétique ne laisse aucun doute, cette dynamique ascendante pose néanmoins problème, dans le sens où elle ne se résume pas à un cheminement du faux prophète vers la conversion mais implique aussi une sorte de sacrement du faux, notamment dans l'Ysengrimus.

\section{Ouverture sur une réflexion spirituelle}

Le problème tient d'abord au fait que la parole du faux prophète ouvre sur une vraie réflexion spirituelle, voire sur une morale chrétienne ${ }^{74}$. Dans la Topographia Hibernica, la rencontre avec le loup débouche ainsi sur un questionnement théologique qui aboutit à l'idée selon laquelle la métamorphose n'a finalement rien de diabolique: en effet, celle-ci s'apparente en réalité à l'un des dogmes essentiels de la religion chrétienne, à savoir la transsubstantiation ou métamorphose du pain et du vin, appelés à signifier le corps et le sang du Christ lors de l'Eucharistie. Cette conclusion, à laquelle parvient le prêtre, dans le processus diégétique, après sa rencontre avec le loup-garou d'Ossory, enjoint le lecteur à reconsidérer la figure de l'hybride, voire à comprendre que celui-ci tire son savoir prophétique de la malédiction dont il est victime et qui lui fait éprouver un autre rapport au monde et une connaissance autre parce qu'animale, sauvage, voire primordiale. En effet, si l'on ajoute au fait que le loup-garou incarne une forme de paganisme, la perception qu'avait la cour anglo-

\footnotetext{
${ }^{74}$ L'argument du Livre VII d'Ysengrimus reste le châtiment des péchés commis par le personnage éponyme tout au long du roman.
} 
normande ${ }^{75}$ de l'Irlandais en tant qu'homme sauvage ${ }^{76}$, «primitif », l'on comprend que le loup-garou d'Ossory n'est peut-être rien de moins qu'une créature «primitive », c'est-à-dire primordiale, à l'instar de Merlin, lui aussi lié au loup, et des animaux les plus anciens du monde ${ }^{77}$. Comme eux, notre loup-garou connaît les mystères de l'univers grâce à son expérience de la métamorphose ${ }^{78}$; mais ce prophète qui parle de saint Natal (†564) et dont le discours s'inspire de l'Ancien Testament ${ }^{79}$ et non du Nouveau, détient aussi les secrets du passé, ce qui fait presque de lui un vestige des temps anciens voire archaïques. D'ailleurs, c'est peut-être ce statut particulier qui lui donne le droit de prophétiser $^{80}$ et fait de lui un vrai prophète converti à la vérité de Dieu, une créature primordiale qui n'a pas seulement l'expérience des anciens temps païens mais aussi celle des

${ }^{75}$ Laquelle constitue l'horizon d'attente du récit.

${ }^{76}$ Joep Leersen, «Wildness, Wilderness, and Ireland: Medieval and Early-Modern Patters in the Demarcation of Civility », Journal of the History of Ideas, 56, 1, janvier 1995, p. 25-39.

${ }^{77}$ Les animaux les plus anciens du Monde sont le merle de Cilgwri, le cerf de Redynvre, le hibou de Kwm Kawlwyt, l'aigle de Gwernabwy et le saumon de Llynn Llyw ( $c f$. Kulhwch et Olwen dans Les Mabinogion du Livre rouge de Hergest, avec les variantes du Livre blanc de Rhydderch, éd. Joseph Loth, Genève, Slatkine, 1975, p. 323-327).

78 Notons d'ailleurs que l'acte de prophétiser est une véritable expérience de métamorphose, selon $1 \mathrm{Sm} \mathrm{X,} 6$ : « et insiliet in te spiritus Domini et prophetabis cum eis et mutaberis in virum alium ». " Et l'esprit de Dieu te saisira, et tu prophétiseras avec eux [la troupe de prophètes de Guibéa-Elohim], et tu te transformeras en un autre homme » (trad. cit., p. 298).

${ }^{79}$ Notons d'ailleurs que l'un des deux livres dont s'inspire la prophétie du loup, l'Ecclésiastique ou Siracide, est considéré par les catholiques comme l'un des livres sapientiaux de l'Ancien Testament, alors que les juifs et les protestants, le considèrent comme apocryphes. Il n'est donc pas anodin qu'une créature aussi ambigüe que le loup-garou, créatures des mânes et des frontières, ne fasse référence à un texte déjà controversé au Moyen Âge.

${ }^{80}$ En amont même des considérations de saint Thomas d'Aquin pour qui la prophétie est avant tout un acte de connaissance: $c f$. Saint Thomas d'Aquin, Summa theologiae, $2^{a}-2^{a e}, 171$ : "Respondeo dicendum quod prophetia primo et principaliter consistit in cognitione "; trad. Henri-Dominique Gardeil dans Somme théologique, Paris, Éditions du Cerf, 1997, p. 966 : «La prophétie est premièrement et principalement un acte de connaissance $»$. 
anciens temps bibliques, et qui symbolise ainsi l'histoire même de l'évangélisation de son peuple ${ }^{81}$.

\section{De la conversion à la subversion : pour un sublime « en faux » dans la prophétie du loup}

Entre conversion et subversion, la prophétie du loup s’épanouit donc dans un fragile entre-deux où le garou, à la manière d'un oracle, prophétise à la demande du prêtre qui pressent que la créature déborde d'un savoir singulier :

Inter ultima vero confabulationis hujus verba, quaesivit presbyter a lupo utrum gens inimica, quae in insulam supervenerat olim, foret diu in ea permansura ${ }^{82}$.

L'on remarque d'ailleurs qu'après cette prophétie le récit s'épuise dans le silence de l'ellipse pour reprendre « deux ans plus tard», comme s'il n'y avait rien à ajouter au discours prophétique, comme si ce dernier se suffisait à lui-même et transcendait le récit à la manière de la parole sublime, celle qui ravit l'âme par un je ne sais quoi et qui, comme le loup, nous laisse le souffle coupé.

La prophétie finale dans l'Ysengrimus a elle aussi quelque chose de sublime, mais un sublime du faux et de la subversion, une terribilitas

${ }^{81}$ L'idée reste implicite dans le texte de la Topographia Hibernica, mais elle est néanmoins latente à la fin de la prophétie du loup-garou (éd. cit., p. 103 : «Quamdiu ergo gens ea mandata Domini custodierit, et in viia ejus ambulaverit, tuta manebit et inconvulsa. Sin autem [...] ad nostros ex convictu mores forte descenderit, divinam in se quoque proculdubio vindictam provocabunt." ("Aussi longtemps que la nation étrangère observera les commandements du Seigneur et marchera dans Ses voies, elle sera en sûreté et restera invincible. Mais si [...] ils en viennent, à notre contact, à adopter nos mœurs dépravées, sur eux aussi sans doute ils attireront la vengeance divine. », trad. cit., p. 213). La mention des « mœurs dépravées » du peuple d'Ossory renvoie en effet à leurs coutumes païennes.

${ }^{82}$ Giraldus Cambrensis, Topographia Hibernica, II, XIX, op. cit., p. 102 ; « Au cours des derniers propos qu'ils échangèrent, le prêtre demanda au loup si la nation ennemie qui était un jour arrivée dans l'île allait y demeurer longtemps. » (trad. cit., p. 213). 
hystérique qui, après que l'avatar du Livre de Paix a été extrait du foie d'Ysengrin, s'abat comme une tempête sur le roman :

Flatibus ergo malis obstacula nulla resistant,

Nec tenui strepitu sibilet aura nocens,

Ut caueant homines et, quem prope leserit aer,

Verberet infidum deuocatque genus! ${ }^{83}$

Le texte se charge d'images frappantes, entre cruauté pure et expiation, vérité du vice et parodie burlesque, didactisme terrible et sadomasochisme. Ainsi en est-il par exemple dans ce passage où est décrit le fléau de la servante qui aura laissé perdre son honneur :

Obsequa si fuerit stirpis quid nacta prophane;

Segnities illam continuata premat,

Nox hiberna breuis misere uideatur, ut orto

Sole ter undecies surgere iussa neget [...]

Brachia tum costaque humerosque et crurafemurque

Timporaque et collum strennuus unguis aret! ${ }^{84}$

Dans ce chaos ${ }^{85}$ violent et bestial où souffle un vent d'horreur, symbolisé par le «longo /eruptu luctans horreat aura foras ${ }^{86}$ » des vers 343-344, le loup paraît couronné d'une aura singulière au moment il prend violemment conscience de son destin, et le ton qu'il emploie change brutalement pour devenir profondément sentencieux: «Mors omnes miscros pensat honesta dies ${ }^{87} »(\mathrm{v} .304-305)$, «Interitum turpem celebris

\footnotetext{
${ }^{83}$ Nivardus, Ysengrimus, op. cit., v. 321-324, p. 385 ; «Aucun obstacle ne résistera donc aux mauvais souffles, et le bruit de la vilaine brise ne sera pas un léger sifflement! Cela pour que les hommes fassent attention, et pour que celui qu'aura offensé une bouffée toute proche, frappe et maudisse l'engeance déloyale!» (trad. cit.).

${ }^{84}$ Ibid., v. 327-336, p. 385-386 ; «Si une servante prend quelque chose de cette race impie, qu'une paresse continuelle s'abatte sur elle : la nuit d'hiver paraitra courte à la malheureuse, si bien qu'à l'aurore, après avoir reçu trente-trois fois l'ordre de se lever, elle refusera toujours de le faire [...]. Un ongle énergique lui laboure alors les bras, les côtés, les épaules, les jambes, les cuisses, les tempes et le cou ! (trad. cit.).

${ }^{85} \mathrm{Cf}$. infra, les vers 354-359 cités dans la note 64.

${ }^{86}$ Éd. cit. « Un bruit d'éruption long et horrifique » (trad. cit).

87 «Une fin honorable rachète tous les jours d'une vie misérable » (trad. cit.).
} 
vindicta secundat ${ }^{88} \gg($ v. 307). Un curieux clair-obscur se dessine en effet autour du loup tandis qu'il prophétise et que l'univers autour de lui sombre dans une apocalypse burlesque, sous l'égide d'Agemundus: «Hoc equidem non est ingens in demone uirtus, /Sed, queccumque potest, perficit absque dolo ${ }^{89} \gg$. Le patronage de ce génie de la subversion, héros de cette maudite prophétie ou prophétie de malédiction, ne sonne-t-il pas comme un aveu de faiblesse de la part du prophète?

Par les mots et les mots seuls, Ysengrin joue de son ambivalence fondamentale, lui qui de faux prophète chrétien devient ainsi vrai prophète du faux, porte-parole non pas tant de Dieu que du deus ex machina à l'œuvre ici, Nivard. Ainsi la prophétie d'Ysengrin débouche-t-elle sur une violente diatribe dirigée contre la papauté et le clergé aux vers 561-619, nourrie du déchaînement apocalyptique des éléments ${ }^{90}$ en forme de visions terribles :

Quid, cum seuierit fornax Chaldea lacusque

Et quem non homines ut timuere fere?

Quid torrens Cison, quid cladis comperit

Endor

Pestibus hic populos, hic cecidisse fame $?^{91}$

La longue énumération, aux vers 67-574, des multiples cataclysmes qui ont frappé l'humanité depuis les temps bibliques, sertie dans une structure anaphorique forte, déferle comme une vague immense sur le texte, traversé par des images violentes qui ne vont pas sans rappeler

88 «Une vengeance éclatante adoucit un trépas infamant» (trad. cit.).

${ }^{89}$ Nivardus, Ysengrimus, op. cit., v. 311-312, p. 384 ; «Sans doute ce démon n'a-t-il pas une énorme puissance, mais il accomplit honnêtement tout ce qui est en son pouvoir » (trad. cit.).

${ }^{90}$ Avec toujours le vent comme fil conducteur de la vision, un élément dont on sait qu'il était lié au loup dans l'imaginaire médiéval puisque depuis Pline l'Ancien, l'on avait coutume de dire que le loup vivait de vent pendant trois mois, chaque année.

${ }^{91}$ Ibid., v. 576-580, p. 397 ; " Pourquoi rappeler le temps où sévissaient en Chaldée la fournaise et la fosse aux lions, pourquoi parler de celui que les bêtes craignirent moins que les hommes ? Pourquoi parler des désastres dont la rivière Kison et la ville d'Endor furent les témoins, tandis que les peuples succombaient ici aux épidémies et là à la faim ? » (trad. cit.). 
l'esthétique de la terribilitas que théoriseront plus tard Addison et Burke. Là, après la mort d'Ysengrin, Nivard prend le relais de son loup ${ }^{92}$ une fois que celui-ci s'est tu pour toujours ou presque ${ }^{93}$, comme pour achever sa prophétie en en extrayant la quintessence, autrement dit cette parole profondément morale bien qu'enrobée d'une enveloppe hallucinée.

Cependant, au-delà du caractère profondément inspiré de la prophétie médiévale en tant que parole divine, l'expression prophétique en elle-même comporte peut-être une part inéluctable de subversion, elle qui se dit le plus souvent par le biais d'une «métaphore cryptique» ou du moins, d'une «formulation ambigüe ${ }^{94}$ », et ne révèle jamais que des «bribes, vraies, sans doutes, mais partielles et partiales, donc potentiellement trompeuses ${ }^{95} »$. Il semblerait donc qu'en dernière instance, les loups de Nivard et de Giraud de Barri nous enjoignent à comprendre que prophétie et fausse prophétie, prophète et faux prophète constituent finalement les deux facettes d'une même vérité.

C'est sur cette idée que s'achève cette étude au cours de laquelle, précisément, deux visages du prophète loup se sont vu confrontés, et avec eux deux prophéties très différentes dont l'une, se réclamant comme fausse, relève de la malédiction, tandis que l'autre tend vers une l'interprétation de l'Histoire, voire vers l'exégèse. À la lecture des œuvres de Giraud de Barri et de Nivard, il apparaît que le loup peut aussi bien incarner le faux prophète que le vrai, en raison de sa qualité d'animal-guide, notamment

\footnotetext{
${ }^{92}$ Dans le cadre du débat final opposant Salaura à Reinardus.

${ }^{93}$ En effet, la toute fin du roman sonne comme un appel de Reinardus à la résurrection d'Ysengrin: "Patrue care, iaces! utinam efficerere superstes », s'exclame-t-il au vers 705 («Mon cher oncle, tu es mort ! S'il plaisait à Dieu de te ressusciter », éd. et trad. cit.).

${ }^{94}$ Richard Traschler, « Moult obscure parleüre : quelques observations sur la prophétie médiévale », dans Moult obscures paroles. Études sur la prophétie médiévale, dir. Richard Trachsler, Paris, Presses de 1'Université Paris-Sorbonne, 2007, p. 8.

${ }^{95}$ Ibid., p. 8.
} 
dans les traditions celtiques qui en ont fait une créature douée d'une connaissance supérieure, un animal à la frontière des deux mondes. En effet, comme beaucoup d'animaux liés à l'univers de la chasse ${ }^{96}$, le loup est traditionnellement considéré comme un animal familier de l'autre monde, au-delà même de sa qualité de prédateur; l'on ne s'étonnera donc pas que dans nos deux textes, la prophétie du loup soit déclenchée par l'imminence de la mort, entre extrême-onction et agonie du prophète. Animal des frontières, des limites et de l'entre-deux, le loup parvient ainsi à convertir voire à subvertir le discours du faux prophète en vérité chrétienne, grâce à son caractère proprement ambivalent, lequel transparaît jusque dans sa parole, tour à tour sacrée ou sacrificatrice. En vertu de sa double nature, à la fois maudite - Ysengrin - et merveilleuse - le garou d'Ossory -, le loup est un outil privilégié de la parole subversive, parole prophétique autant que satirique: en effet, il est surtout le chantre des vérités troublantes, notamment lorsque le fait de dire la vérité revient à dénoncer toute forme de corruption, a fortiori ce clergé renégat contre lequel maître Nivard prend la plume comme d'autres prendraient les armes. Ainsi le loup se fait-il messager non pas tant du Dieu créateur mais de son relais ici-bas, le clerc, l'auctoritas, l'auteur dont l'animal constitue le double littéraire, pour devenir soit un guide et un moteur de réflexion pour Giraud de Barri, soit un souffre-douleur en forme d'expédient chez Nivard, porte-voix de sa parole empêchée parce que dérangeante, hologramme subtil à travers lequel filtre une vision dédoublée du clergé contemporain. C'est d'ailleurs grâce à cette complicité que le faux prophète, en faisant fusionner vraie et fausse prophétie dans sa gueule d'enfer, ouvre la voie à la vérité tout en faisant prendre conscience au lecteur de la part de subversion propre à la parole

\footnotetext{
${ }^{96}$ À l'instar du cerf, de la biche et du sanglier qui sont tous des animaux psychopompes dans l'imaginaire médiéval. L'on a d'ailleurs pu voir que dans la Topographia Hibernica comme dans des textes plus proprement narratifs tels que le roman Guillaume de Palerne, le loup joue le rôle de guide dans la trame du récit.
} 
prophétique, parole de l'entre-deux qui fait du loup un support original de la satire, dont la folie inspirée se trouve au cœur des enjeux liés à la prophétie médiévale. Au-delà de sa fonction de dévoilement, à l'instar du loup, la prophétie médiévale garde ainsi jalousement un peu de son mystère, elle qui, comme la bête, ne saurait se laisser enfermer dans une stricte définition mais renferme un savoir dont on aime se dire qu'il conserve une part d'inaccessible, comme une parole sauvage difficilement saisissable mais qui continue d'aiguiser la curiosité du lecteur. 